\title{
Single molecule kinetics of horseradish peroxidase exposed in large arrays of femtoliter-sized fused silica chambers $\dagger$
}

Cite this: Analyst, 2013, 138, 4260

Received 22nd April 2013

Accepted 16th May 2013

DOI: 10.1039/c3an00809f

\author{
Benno N. Ehrl, Raphaela B. Liebherr and Hans H. Gorris*
}

www.rsc.org/analyst

Large arrays of femtoliter-sized chambers were etched into the surface of fused silica slides to enclose and observe hundreds of single horseradish peroxidase (HRP) molecules in parallel. Individual molecules of HRP oxidize the fluorogenic substrate Amplex Red to fluorescent resorufin in separate chambers, which was monitored by fluorescence microscopy. Photooxidation of Amplex Red and photobleaching of resorufin have previously limited the analysis of HRP in femtoliter arrays. We have strongly reduced these effects by optimizing the fluorescence excitation and detection scheme to yield accurate single molecule substrate turnover rates. We demonstrate the presence of long-lived kinetic states of single HRP molecules that are individually different for each molecule in the array. The large number of molecules investigated in parallel provides excellent statistics on the activity distribution in the enzyme population, which is similar to that reported for other enzymes such as $\beta$-galactosidase. We have further confirmed that the product formation of HRP in femtoliter chambers is 10 -fold lower than that in the bulk solution due to the particular two-step redox reaction mechanism of HRP.

\section{Introduction}

Single molecule studies have unravelled different conformational states of individual enzyme molecules ${ }^{1}$ that lead to substrate turnover fluctuations in subsequent catalytic cycles (dynamic heterogeneity) ${ }^{2-5}$ or broad and long-lived activity distributions in an enzyme population (static heterogeneity). ${ }^{6-8}$ Static heterogeneity has been investigated in large arrays of femtoliter $\left(\mu \mathrm{m}^{3}\right)$-sized chambers etched into the end of glassoptical fibre bundles, ${ }^{9}$ into the surface of fused silica slides, ${ }^{10}$ or moulded into polydimethylsiloxane (PDMS). ${ }^{11}$ Femtoliter arrays confer several advantages because (1) no surface

Institute of Analytical Chemistry, Chemo- and Biosensors, University of Regensburg, D-93040 Regensburg, Germany. E-mail: hans-heiner.gorris@ur.de; Fax: +49-941943-4064

$\dagger$ Electronic supplementary information (ESI) available: Two additional graphs and a movie. See DOI: 10.1039/c3an00809f immobilization of enzymes is required, which may affect their conformation and activity, (2) surface etching yields large arrays of several thousand femtoliter chambers with a well-defined size and volume, and (3) hundreds of individual enzyme molecules can be observed simultaneously, which allows for an excellent statistical analysis of the kinetics in an enzyme population. ${ }^{12}$ Despite these advantages, only a few model enzymes that are stable and display high substrate turnover rates have been investigated in femtoliter arrays so far, e.g. lactate dehydrogenase, ${ }^{10} \beta$-galactosidase, ${ }^{7,13}$ chymotrypsin ${ }^{14}$ and horseradish peroxidase (HRP). ${ }^{15}$ The monomeric enzyme HRP is a particularly interesting subject for single molecule studies because the heterogeneity in its activity can be attributed to the conformation of a single catalytic site.

HRP was previously studied at the single molecule level in glass-optical fibre bundles using the substrate $N$-acetyl-3,7dihydroxyphenoxazine (Amplex Red). ${ }^{11,15}$ Amplex Red is one of the most stable and sensitive fluorogenic substrates that is oxidized to highly fluorescent resorufin by HRP. ${ }^{16,17}$ According to the catalytic cycle outlined in Fig. 1, the heme prosthetic group of HRP undergoes a three-step redox cycle. ${ }^{18}$ Hydrogen peroxide $\left(\mathrm{H}_{2} \mathrm{O}_{2}\right)$ oxidizes the ground state of the heme group to Compound I in a conventional two-electron transfer step. The reduction of Compound I to restore the ground state, in contrast, involves two subsequent one-electron transfer steps from two Amplex Red molecules and the formation of Compound II as a reaction intermediate. ${ }^{19,20}$ In these steps, two non-fluorescent radical molecules of Amplex Red per $\mathrm{H}_{2} \mathrm{O}_{2}$ molecule are generated that subsequently undergo a dismutation reaction to form one molecule of resorufin and one molecule of Amplex Red.

The $\mathrm{H}_{2} \mathrm{O}_{2}$ consumption and resorufin production can deviate from a $1: 1$ stoichiometry if competing reactions lead to the formation of products other than resorufin. ${ }^{21}$ Such competing reactions can be observed if the concentration of Amplex Red is very low ( $<50 \mathrm{pM}$ ) or if the reaction is performed in a very small confinement where the large surface-to-volume ratio may lead to surface-mediated side reactions of Amplex Red 


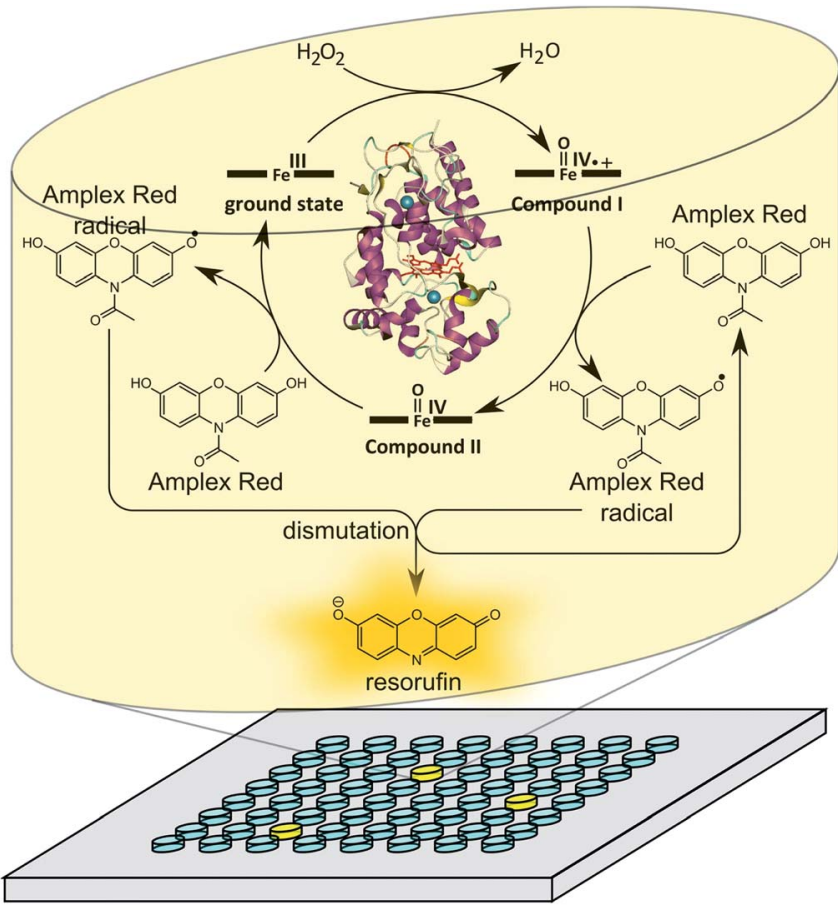

Fig. 1 Substrate turnover of single HRP molecules in a large array of femtoliter chambers. HRP catalyzes the formation of non-fluorescent Amplex Red radicals that undergo a dismutation reaction before highly fluorescent resorufin is generated. The concentration of HRP is adjusted to a ratio of one molecule in every twentieth chamber such that, according to Poisson statistics, not more than a single molecule is present in any chamber. The femtoliter chambers are tightly sealed under mechanical pressure (not shown) and the product resorufin accumulates in chambers containing a single molecule of HRP, which is monitored in parallel by wide-field fluorescence microscopy.

radicals. For example, the apparent substrate turnover of single HRP molecules in a femtoliter chamber ${ }^{15}$ was only $10 \%$ and in an attoliter liposome ${ }^{22}$ even less than $1 \%$ of the bulk substrate turnover. Another problem of probing single HRP molecules in femtoliter arrays is the need for strong excitation light, which induces autocatalytically enhanced photooxidation of Amplex Red in the presence of trace amounts of resorufin ${ }^{23}$ and leads to a high fluorescence background increase. ${ }^{15}$ Here, we have devised a new type of femtoliter array on the surface of fused silica slides to improve the fluorescence signal acquisition under milder excitation conditions.

\section{Experimental}

\section{Buffers and reagents}

Phosphate buffered saline (PBS) (136 mM NaCl, $8.1 \mathrm{mM}$ $\mathrm{Na}_{2} \mathrm{HPO}_{4}, 2.7 \mathrm{mM} \mathrm{KCl}$ and $1.5 \mathrm{mM} \mathrm{KH} \mathrm{PO}_{4}$ ) was prepared and pH-adjusted to 7.4 by $1 \mathrm{M} \mathrm{HCl}$. Lyophilized, salt free horseradish peroxidase (HRP, EC number: 232-668-6, RZ $\geq 3.0$ ) purchased from Sigma (http:/www.sigmaaldrich.com) was reconstituted to $100 \mu \mathrm{M}$ in $50: 50$ PBS-glycerol, divided into aliquots and stored at $-20{ }^{\circ} \mathrm{C}$. A $20 \mathrm{mM}$ stock solution of Amplex Red (Life Technologies, http:/www.lifetechnologies. com) in dimethyl sulfoxide (DMSO) was split into aliquots and stored at $-20{ }^{\circ} \mathrm{C}$. A $10 \mathrm{mM}$ stock solution of resorufin (Life
Technologies) in DMSO was aliquoted and stored at $-20{ }^{\circ} \mathrm{C}$. The concentration of $\mathrm{H}_{2} \mathrm{O}_{2}$ was determined in a spectrometer at $240 \mathrm{~nm}$ using a molar extinction coefficient of $43.6 \mathrm{M}^{-1} \mathrm{~cm}^{-1} .^{24}$ The single molecule experiments in femtoliter arrays were carried out at room temperature in PBS containing $0.05 \mathrm{mg}$ $\mathrm{mL}^{-1}$ of bovine serum albumin (Sigma) and $0.2 \mathrm{mg} \mathrm{mL}^{-1}$ of Tween 20 (Sigma), which served to minimize non-specific surface binding. ${ }^{25}$ The stock solutions were diluted just prior to experimentation.

\section{Preparation of femtoliter array and gasket}

Four-inch fused silica wafers with a thickness of $0.5 \mathrm{~mm}$ were purchased from siegert (http://www.siegertwafer.de) and microstructured by photolithography and reactive ion etching. Arrays of $250 \times 250=62500$ cylindrical wells (diameter: 4.2 $\mu \mathrm{m}$; depth: $3 \mu \mathrm{m}$; pitch: $10 \mu \mathrm{m}$ ) with an edge length of $2.5 \times$ $2.5 \mathrm{~mm}$ were etched into the surface of the fused silica wafer. The exact chamber size was determined to be $41 \mathrm{fL}$ by scanning electron microscopy. The fused silica wafer was subsequently cut into slides of $15 \times 15 \mathrm{~mm}$ such that the array was positioned in the centre of each slide. The femtoliter arrays were used repeatedly and cleaned between measurements by using Piranha solution (1:3 mixture of $30 \% \mathrm{H}_{2} \mathrm{O}_{2}$ and concentrated $\mathrm{H}_{2} \mathrm{SO}_{4}$ ) and washing with bidistilled water under ultrasonication. A gasket for array sealing was made of PDMS (SYLGARD 184 silicone elastomer kit, Dow Corning, http:// www.dowcorning.com). The PDMS was cast on a clean and smooth surface to a height of $0.5 \mathrm{~mm}$, polymerized at $37^{\circ} \mathrm{C}$ for $48 \mathrm{~h}$ and cut into pieces of $5 \times 5 \mathrm{~mm}$. Before single use as a gasket, the PDMS pieces were cleaned with curd soap and rinsed with bidistilled water.

\section{Enclosing single molecules of HRP in femtoliter arrays}

The dry femtoliter array was mounted on a custom-built array holder on an inverted epi-fluorescence microscope (Eclipse TiE, Nikon, http://www.nikoninstruments.com). Just prior to each measurement, a solution of 1.8 pM HRP, $250 \mu \mathrm{M}$ Amplex Red and varying concentrations of $\mathrm{H}_{2} \mathrm{O}_{2}$ was prepared. The wells were filled by dispensing $5 \mu \mathrm{L}$ of this solution on top of the femtoliter array. The PDMS gasket was pressed by mechanical pressure against the surface of the array to form separate femtoliter chambers that contained individual HRP molecules and to prevent diffusion of the reaction product into neighboring chambers. Image acquisition was started within one minute after mixing enzyme and substrate.

\section{Monitoring the substrate turnover of single enzyme molecules by fluorescence microscopy}

Images were acquired through the opposite face of the femtoliter array by wide-field fluorescence microscopy on the inverted epi-fluorescence microscope equipped with a pre-centred fiberoptical mercury lamp (Intensilight, Nikon), a $20 \times$ objective $(\mathrm{NA}=0.75)$, a filter set with $\lambda_{\mathrm{ex}}=577 \mathrm{~nm}$ and $\lambda_{\mathrm{em}}=595 \mathrm{~nm}$ (Chroma Technology, http://www.chroma.com), and a sensitive sCMOS camera (5.5 megapixels, Andor Technology, http:// www.andor.com). Images were taken every $30 \mathrm{~s}$ for $10 \mathrm{~min}$ 
using an exposure time of $1 \mathrm{~s}$ under low excitation illumination (neutral density filter $=32$ ). Standard imaging software (NISElements, Nikon) was used for controlling image acquisition, assigning the fluorescence signals in individual chambers, and displaying the fluorescence intensity in pseudocolour. The fluorescence signals were background-corrected by subtracting the signals of empty chambers and calibrated by enclosing standard solutions of resorufin in the femtoliter array. The signal increase over the first 6 min of each measurement was used for calculating apparent substrate turnover rates.

\section{Results and discussion}

\section{Requirements for analyzing the substrate turnover of individual HRP molecules}

As single enzyme molecules cannot be deposited individually into femtoliter chambers, the single molecule approach presented here relies on a random distribution of enzyme molecules in the array. When the enzyme concentration is lowered to $36 \mathrm{pM}$ and this solution is distributed on an array of chambers defining a volume of $41 \mathrm{fL}$, there is on average one enzyme molecule present per chamber $(a=1)$, and the probability $P_{a}$ of finding $n$ enzyme molecules in any given chamber can be calculated by the Poisson distribution: ${ }^{12}$

$$
P_{a}(n)=\frac{e^{-a} \times a^{n}}{n !}
$$

At this ratio, however, some chambers also contain zero and others more than a single enzyme molecule. Thus, the enzyme concentration must be further reduced to ensure that the substrate turnover in a chamber relates to the activity of a single enzyme molecule only. At a twenty-fold lower enzyme concentration of $1.8 \mathrm{pM}(a=0.05)$, most of the chambers are empty $\left(P_{0.05}(0)=95 \%\right), 5 \%$ contain a single enzyme molecule only $\left(P_{0.05}(1)=5 \%\right)$, and the probability that a well contains more than a single enzyme molecule is negligibly low $\left(P_{0.05}(>1)=\right.$ $0.1 \%)$. Although the majority of chambers are empty, there are still a few hundred chambers available for single molecule analysis because typically 5000 chambers are in the field of view of the microscope. Counting revealed that $238 \pm 7$ (average of three measurements and standard deviation) of 5000 chambers were active $(a=0.048)$, i.e. contained a single molecule of HRP. ${ }^{26}$

The concentrations of Amplex Red and $\mathrm{H}_{2} \mathrm{O}_{2}$ were chosen $10^{8}$-fold higher than HRP such that each chamber contained the same amount of substrate. The maximum solubility of Amplex Red in water is $300 \mu \mathrm{M}^{\mathbf{1 6}}$ and a concentration of $250 \mu \mathrm{M}$ was used in all experiments, which is equivalent to about six million molecules of Amplex Red in a volume of $41 \mathrm{fL}$. Consequently, less than 5\% of Amplex Red was consumed over a time course of 6 min considering a maximal substrate turnover rate of $740 \mathrm{~s}^{-1} \cdot{ }^{15}$ During the same time, an initial concentration of 63 $\mu \mathrm{M} \mathrm{H}_{2} \mathrm{O}_{2}$ decreased by $20 \%$ only. Lower concentrations of $\mathrm{H}_{2} \mathrm{O}_{2}$, however, were not used to avoid substrate depletion. Given these parameters, the femtoliter wells had the optimal size to avoid substrate depletion but to allow for the accumulation of a detectable concentration of the product resorufin.

\section{Optimization of image acquisition}

Although HRP exhibits a high substrate turnover rate, only $10 \%$ of the substrate turnover yielded the fluorescent product resorufin when the reaction was performed in femtoliter chambers located on one end of a glass-optical fibre bundle. ${ }^{15}$ Due to the limited acceptance angle of the optical waveguides connected to each chamber, it was necessary to apply strong excitation light to efficiently record the product formation of single HRP molecules. Strong excitation light, however, led to photooxidation of Amplex Red, ${ }^{23}$ which increased the fluorescence background, and photobleaching of the product resorufin., ${ }^{7,13}$

Here, we have optimized the optical path as well as the detection system to allow for employing milder excitation conditions. A femtoliter array etched into the surface of a fused silica slide was directly monitored under a high resolution objective. A very high numerical aperture, however, is only available when using a high magnification at the expense of a smaller field of view, i.e. fewer chambers that can be monitored simultaneously. To account for both a relatively high numerical aperture as well as a large field of view (5000 chambers) we chose a $20 \times$ objective with a numerical aperture of 0.75 to collect the emission light efficiently.

For single molecule detection of fluorophores by wide-field microscopy, typically ICCD or EMCCD (intensified/electron multiplying charged-coupled device) cameras are employed due to their very high sensitivity. The sensitivity of the camera, however, is not the only limiting factor when monitoring the product formation of single HRP molecules in femtoliter chambers because thousands of fluorescent product molecules accumulate in the chambers. Rather, a high dynamic range and a high resolution are required to account for a strong fluorescence signal increase and for resolving 5000 chambers under the field of view. The signal amplification of ICCD and EMCCD cameras, however, results in a lower dynamic range, and their resolution is low compared to CCD (charged-coupled device) or CMOS (complementary metal oxide semiconductor) cameras. Modern CMOS cameras, in contrast, combine high sensitivity, high dynamic range and high resolution and thus were chosen for monitoring single molecules of HRP in femtoliter arrays. To demonstrate the performance of our single molecule detection platform, we enclosed a solution containing only $1 \mu \mathrm{M}$ of the fluorescent product resorufin in the femtoliter array. The fluorescence signal decreased by less than $1 \%$ due to photobleaching when images were taken over a time course of $10 \mathrm{~min}$ (ESI $\dagger$ ). Consequently, it is not necessary to correct the time traces of individual enzyme molecules for photobleaching., ${ }^{7,13}$

The image acquisition was further optimized by adjusting the excitation mode. According to classical linear optics the total fluorescence signal does not change when the exposure time is increased by a certain factor and the excitation intensity is reduced by the same factor. The intensity of the excitation light, however, leads to much stronger photooxidation of the substrate Amplex Red relative to the exposure time. ${ }^{23}$ Thus, the photooxidation of Amplex Red in the femtoliter chambers was strongly reduced by minimizing the intensity of the excitation light (ND filter $=32$ ) and increasing the exposure time to $1 \mathrm{~s}$. 

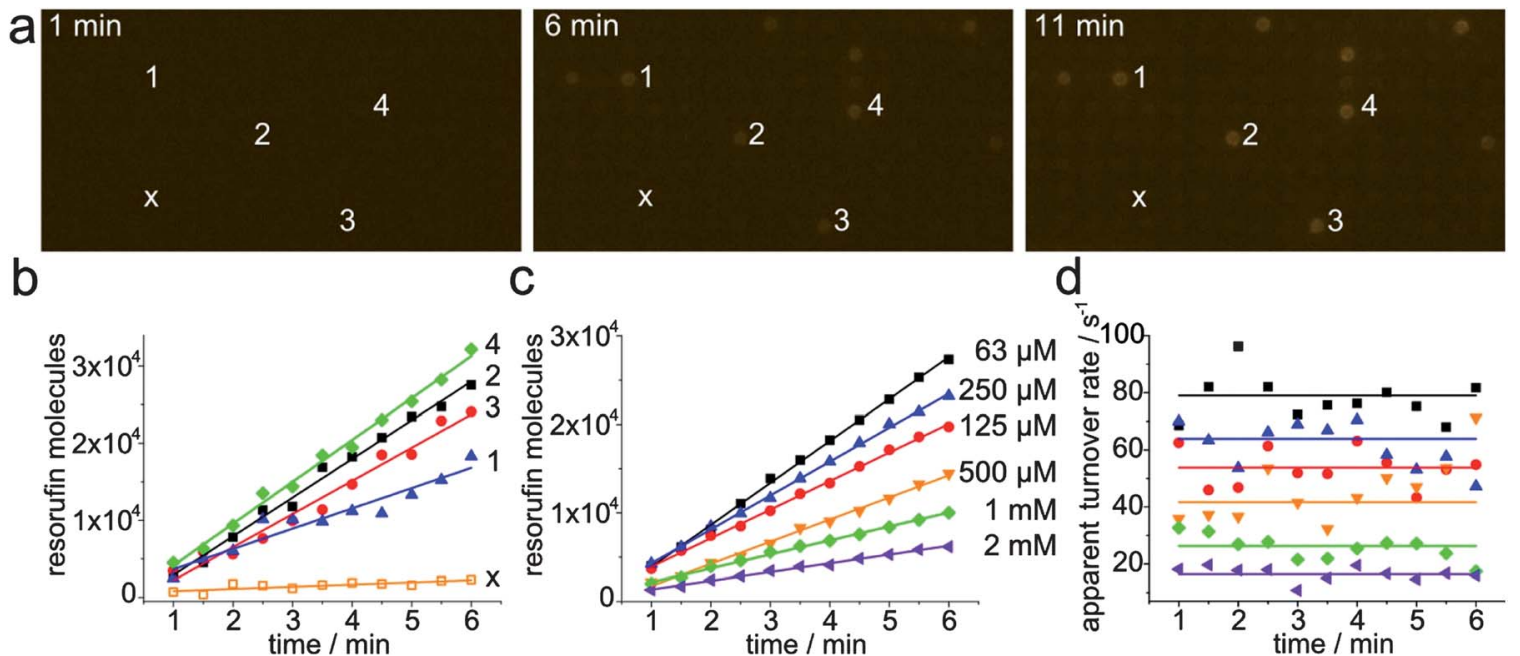

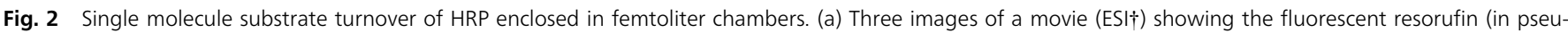

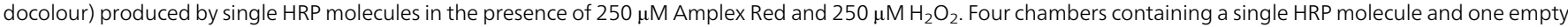

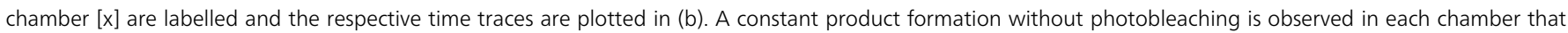

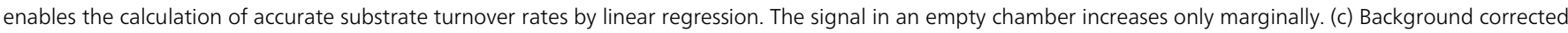

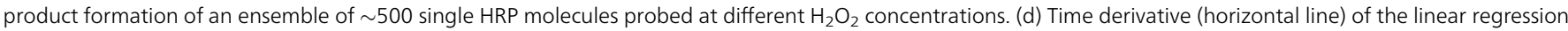
shown in (c). Fluctuations are calculated as $\left(N_{t}-N_{t-60 \mathrm{~s}}\right) / 60 \mathrm{~s}$, where $N$ denotes the signal intensity at a given time point $(t)$.

Standard solutions of resorufin were enclosed in the femtoliter array to calibrate the fluorescence signal intensities under the optimized excitation conditions. The fluorescence intensity increases linearly with the concentration of resorufin in the chambers (ESI $\dagger$ ). The concentration of resorufin is further indicated as the total number of resorufin molecules in the femtoliter volume.

\section{Single molecule kinetics of HRP}

When images of the enzyme reaction were recorded every $30 \mathrm{~s}$, the fluorescence signal in every twentieth chamber increased linearly for more than 6 min after starting the measurement. To account for the enzyme independent step involved in the product formation, the activity of individual HRP molecules is denoted as "apparent" substrate turnover rates. Apparent turnover rates were calculated from the slope of the first 11 images. Fig. 2 shows a small section of images taken after $1 \mathrm{~min}$ (the time needed to close the array and start the measurement), $6 \mathrm{~min}$ and $11 \mathrm{~min}$. Although the activity of different HRP molecules is broadly distributed, the fluorescence increase originating from a single HRP molecule is constant and enables the calculation of accurate substrate turnover rates. The fluorescence intensity in the empty chamber, in contrast, increases only marginally and was used for background correction in further data analysis. The average substrate turnover rate of an ensemble of approximately 500 HRP molecules analyzed at various $\mathrm{H}_{2} \mathrm{O}_{2}$ concentrations is summarized in Fig. $2 \mathrm{c}$ and $\mathrm{d}$. HRP displays the highest activity at concentrations between 63 $\mu \mathrm{M}$ and $250 \mu \mathrm{M} \mathrm{H} \mathrm{H}_{2} \mathrm{O}_{2}$ but the activity decreases at higher concentrations because $\mathrm{H}_{2} \mathrm{O}_{2}$ is also an inhibitor of HRP. ${ }^{27,28}$ These data confirm previous reports on the $\mathrm{H}_{2} \mathrm{O}_{2}$-dependent turnover of Amplex Red by HRP at the bulk as well as the single molecule levels. ${ }^{15,17}$ Furthermore, the 10-fold lower apparent substrate turnover rate of single HRP molecules observed in arrays on glass-optical fiber bundles ${ }^{15}$ is also observed in the present study. In both types of femtoliter arrays, the chamber size of 40-50 fL defines the same surface area implicated in potential side reactions of Amplex Red radicals.

Fig. 3 shows the substrate turnover rates of approximately 500 individual HRP molecules from three measurements per concentration of $\mathrm{H}_{2} \mathrm{O}_{2}$ that follow a Gaussian distribution. A coefficient of variation of 30 to $50 \%$ was calculated for concentrations of 63 to $250 \mu \mathrm{M} \mathrm{H}_{2} \mathrm{O}_{2}$, which increased at higher

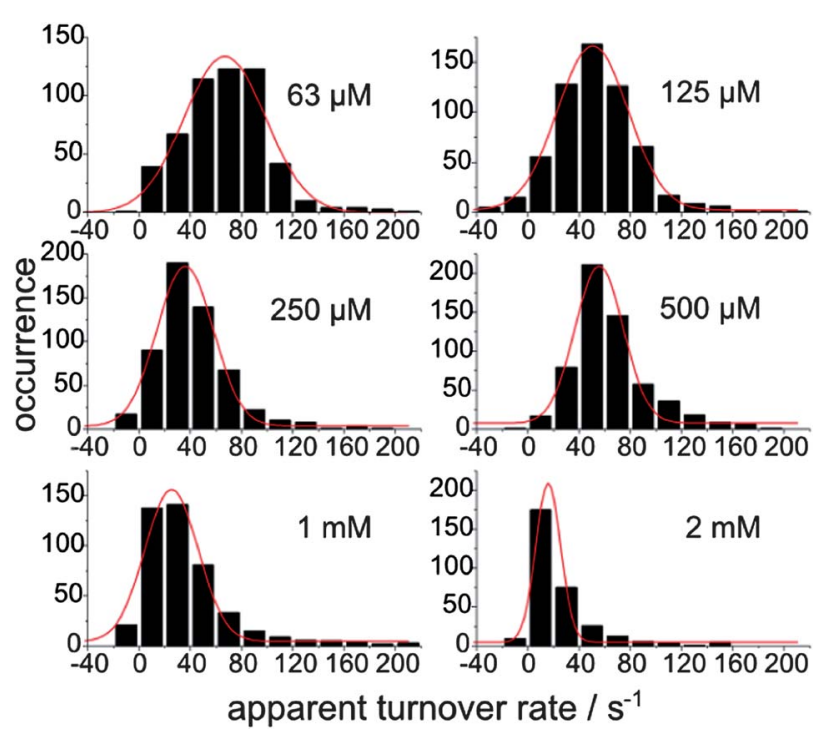

Fig. 3 Apparent substrate turnover distribution of several hundred single HRP molecules probed in the presence of $250 \mu \mathrm{M}$ Amplex Red and various concentrations of $\mathrm{H}_{2} \mathrm{O}_{2}$ (indicated in the graphs). The turnover rates were calculated as described in Fig. 2 . The red curve shows a Gaussian fit of the activity distribution. 
concentrations of $\mathrm{H}_{2} \mathrm{O}_{2}$ (ESI $\dagger$ ). Remarkably, this activity distribution determined in femtoliter arrays etched into the surface of fused silica slides is more than a factor of two narrower than that reported earlier ${ }^{\mathbf{1 5}}$ because the optimized microscopic setup allowed us to obtain more accurate substrate turnover rates over a longer period of time.

The activity distribution indicates the maximally possible conformational heterogeneity in a population of individual HRP molecules because there is an enzyme-independent step involved in the formation of resorufin that may contribute to the heterogeneity in the fluorescence increase. Despite this enzyme-independent step, the single molecule activity distribution of the monomeric enzyme HRP is comparable to that of the tetrameric enzyme $\beta$-galactosidase, which was reported to be $30 \%$ using various substrate concentrations. ${ }^{7}$ The narrower activity distribution of HRP exposed in our new femtoliter array format is expected because the conformational heterogeneity of the monomeric enzyme HRP is related to a single catalytic site. Furthermore, the substrate turnover is primarily mediated by the rigid heme prosthetic group of HRP and not by the conformationally more flexible peptide structure as in the case of $\beta$-galactosidase.

\section{Conclusions}

Femtoliter arrays etched into the surface of fused silica slides constitute a highly sensitive platform for monitoring the substrate turnover of hundreds of single HRP molecules simultaneously. The single molecule analysis of HRP in femtoliter arrays was previously limited by fluctuations in the fluorescence signal due to photooxidation of Amplex Red and photobleaching of resorufin. With the optimized array readout, accurate substrate turnover rates were calculated that were constant over time but differed among individual HRP molecules (static heterogeneity).

\section{Acknowledgements}

We acknowledge funding by the German Research Council (DFG grant GO 1968/3-1) and thank Prof. Helmut Hummel and Albert Hutterer (Regensburg University of Applied Sciences) for preparing microstructured fused silica slides.

\section{Notes and references}

1 V. I. Claessen, H. Engelkamp, P. C. M. Christianen, J. C. Maan, R. J. M. Nolte, K. Blank and A. E. Rowan, Single-biomolecule kinetics: the art of studying a single enzyme, Annu. Rev. Anal. Chem., 2010, 3, 319-340.

2 B. P. English, W. Min, A. M. van Oijen, K. T. Lee, G. Luo, H. Sun, B. J. Cherayil, S. C. Kou and X. S. Xie, Everfluctuating single enzyme molecules: Michaelis-Menten equation revisited, Nat. Chem. Biol., 2006, 2, 87-94.

3 O. Flomenbom, K. Velonia, D. Loos, S. Masuo, M. Cotlet, Y. Engelborghs, J. Hofkens, A. E. Rowan, R. J. Nolte, M. Van der Auweraer, F. C. de Schryver and J. Klafter, Stretched exponential decay and correlations in the catalytic activity of fluctuating single lipase molecules, Proc. Natl. Acad. Sci. U. S. A., 2005, 102, 2368-2372.

4 H. P. Lu, L. Xun and X. S. Xie, Single-molecule enzymatic dynamics, Science, 1998, 282, 1877-1882.

5 K. Velonia, O. Flomenbom, D. Loos, S. Masuo, M. Cotlet, Y. Engelborghs, J. Hofkens, A. E. Rowan, J. Klafter, R. J. M. Nolte and F. C. de Schryver, Single-enzyme kinetics of CALB-catalyzed hydrolysis, Angew. Chem., Int. Ed., 2005, 44, 560-564.

6 Q. Xue and E. S. Yeung, Differences in the chemical reactivity of individual molecules of an enzyme, Nature, 1995, 373, 681-683.

7 D. M. Rissin, H. H. Gorris and D. R. Walt, Distinct and longlived activity states of single enzyme molecules, J. Am. Chem. Soc., 2008, 130, 5349-5353.

8 D. B. Craig, J. T. Nachtigall, H. L. Ash, G. K. Shoemaker, A. C. Dyck, T. M. Wawrykow and H. L. Gudbjartson, Differences in the average single molecule activities of E. coli beta-galactosidase: effect of source, enzyme molecule age and temperature of induction, J. Protein Chem., 2003, 22, 555-561.

9 H. H. Gorris, T. M. Blicharz and D. R. Walt, Optical-fiber bundles, FEBS J., 2007, 274, 5462-5470.

$10 \mathrm{~W}$. H. Tan and E. S. Yeung, Monitoring the reactions of single enzyme molecules and single metal ions, Anal. Chem., 1997, 69, 4242-4248.

11 Y. Rondelez, G. Tresset, K. V. Tabata, H. Arata, H. Fujita, S. Takeuchi and H. Noji, Microfabricated arrays of femtoliter chambers allow single molecule enzymology, Nat. Biotechnol., 2005, 23, 361-365.

$12 \mathrm{H}$. H. Gorris and D. R. Walt, Analytical chemistry on the femtoliter scale, Angew. Chem., Int. Ed., 2010, 49, 3880-3895.

13 H. H. Gorris, D. M. Rissin and D. R. Walt, Stochastic inhibitor release and binding from single-enzyme molecules, Proc. Natl. Acad. Sci. U. S. A., 2007, 104, 1768017685.

14 A. Y. Chen, A. S. Jani, L. Zheng, P. J. Burke and J. P. Brody, Microfabricated arrays of cylindrical wells facilitate singlemolecule enzymology of alpha-chymotrypsin, Biotechnol. Prog., 2009, 25, 929-937.

$15 \mathrm{H}$. H. Gorris and D. R. Walt, Mechanistic aspects of horseradish peroxidase elucidated through single-molecule studies, J. Am. Chem. Soc., 2009, 131, 6277-6282.

16 M. J. Zhou, Z. J. Diwu, N. Panchuk-Voloshina and R. P. Haugland, A stable nonfluorescent derivative of resorufin for the fluorometric determination of trace hydrogen peroxide: applications in detecting the activity of phagocyte NADPH oxidase and other oxidases, Anal. Biochem., 1997, 253, 162-168.

17 V. Towne, M. Will, B. Oswald and Q. J. Zhao, Complexities in horseradish peroxidase-catalyzed oxidation of dihydroxyphenoxazine derivatives: appropriate ranges for $\mathrm{pH}$ values and hydrogen peroxide concentrations in quantitative analysis, Anal. Biochem., 2004, 334, 290296.

18 G. I. Berglund, G. H. Carlsson, A. T. Smith, H. Szoke, A. Henriksen and J. Hajdu, The catalytic pathway of 
horseradish peroxidase at high resolution, Nature, 2002, 417, 463-468.

19 J. N. Rodriguez-Lopez, M. A. Gilabert, J. Tudela, R. N. F. Thorneley and F. Garcia-Canovas, Reactivity of horseradish peroxidase compound II toward substrates: kinetic evidence for a two-step mechanism, Biochemistry, 2000, 39, 13201-13209.

20 J. N. Rodriguez-Lopez, D. J. Lowe, J. Hernandez-Ruiz, A. N. P. Hiner, F. Garcia-Canovas and R. N. F. Thorneley, Mechanism of reaction of hydrogen peroxide with horseradish peroxidase: identification of intermediates in the catalytic cycle, J. Am. Chem. Soc., 2001, 123, 1183811847.

21 J. V. Rodrigues and C. M. Gomes, Enhanced superoxide and hydrogen peroxide detection in biological assays, Free Radical Biol. Med., 2010, 49, 61-66.

22 H. M. Piwonski, M. Goomanovsky, D. Bensimon, A. Horovitz and G. Haran, Allosteric inhibition of individual enzyme molecules trapped in lipid vesicles, Proc. Natl. Acad. Sci. U. S. A., 2012, 109, E1437-E1443.
23 B. Zhao, F. A. Summers and R. P. Mason, Photooxidation of Amplex red to resorufin: implications of exposing the Amplex red assay to light, Free Radical Biol. Med., 2012, 53, 1080-1087.

24 R. W. Noble and Q. H. Gibson, The reaction of ferrous horseradish peroxidase with hydrogen peroxide, J. Biol. Chem., 1970, 245, 2409-2413.

25 K. E. Sapsford and F. S. Ligler, Real-time analysis of protein adsorption to a variety of thin films, Biosens. Bioelectron., 2004, 19, 1045-1055.

26 D. M. Rissin and D. R. Walt, Digital concentration readout of single enzyme molecules using femtoliter arrays and Poisson statistics, Nano Lett., 2006, 6, 520-523.

27 D. Keilin and T. Mann, On the haematin compound of peroxidase, Proc. R. Soc. London, Ser. B, 1937, 122, 119-133. 28 J. B. Wittenberg, R. W. Noble, B. A. Wittenberg, E. Antonini, M. Brunori and J. Wyman, Studies on equilibria and kinetics of reactions of peroxidase with ligands. 2. Reaction of ferroperoxidase with oxygen, J. Biol. Chem., 1967, 242, 626634. 